This item was submitted to Loughborough's Research Repository by the author.

Items in Figshare are protected by copyright, with all rights reserved, unless otherwise indicated.

\title{
Examining the development environments of elite English football academies: The players' perspective
}

PLEASE CITE THE PUBLISHED VERSION

http://dx.doi.org/10.1260/1747-9541.9.6.1457

\section{PUBLISHER}

SAGE @ Multi-Science Publishing

VERSION

AM (Accepted Manuscript)

\section{PUBLISHER STATEMENT}

This work is made available according to the conditions of the Creative Commons Attribution-NonCommercialNoDerivatives 4.0 International (CC BY-NC-ND 4.0) licence. Full details of this licence are available at: https://creativecommons.org/licenses/by-nc-nd/4.0/

\section{LICENCE}

CC BY-NC-ND 4.0

\section{REPOSITORY RECORD}

Mills, Andrew, Joanne Butt, lan Maynard, and Chris G. Harwood. 2019. "Examining the Development Environments of Elite English Football Academies: The Players' Perspective". figshare. https://hdl.handle.net/2134/20798. 
Examining the development environments

1

2

3

4

5

6

7

8

9 Examining the development environments of elite English football academies: The players'

10 perspective

11

12 Andrew Mills, Joanne Butt, and Ian Maynard

13 Centre for Sport and Exercise Science, Sheffield Hallam University, Sheffield, UK

14 Chris Harwood

15 School of Sport, Exercise \& Health Sciences, Loughborough University, UK 


\section{$1 \quad$ ABSTRACT}

2 As a preliminary investigation, we examined elite youth football academy players'

3 perceptions of the quality of their development environment, at a crucial stage in their

4 progression to the professional level. With institutional ethics approval, the Talent

5 Development Environment Questionnaire (TDEQ) [1] was used to survey 50 elite players

6 aged 16-18 ( $m$ 17.1, $\pm s=0.6$ years) recruited from the academies of Premier League and

7 Championship clubs in England. Overall, the results suggest that elite player development

8 environments are perceived to be of a good quality. However, while academies appeared

9 strong in areas related to coaching, organisation, and sport-related support; they were

10 somewhat deficient in areas related to athlete understanding, links to senior progression, and

11 key stakeholder relationships. In addition to the importance of establishing well-integrated

12 youth and senior teams and positive working relationships with parents; the findings

13 underline the necessity for academies to pay close attention to the psychosocial environments

14 they create for developing players. Theoretical considerations and applied implications for

15 those involved in elite player development are discussed.

16 
Examining the development environments

\section{INTRODUCTION}

The development of gifted young football players is paramount on the agenda for those responsible for governing professional football in England (e.g., The Football Association, Premier League). While the English Premier League (EPL) has enjoyed exponential growth over the last two decades, concerns have been raised about the decreasing number of English players that have been developed during this period. Supporting this notion, recent research from the Centre International d'Etude du Sport (CIES) Football Observatory revealed that the playing time of English under-21s in the EPL has fallen to its lowest level [2]. More precisely, in the 2012/13 season, only 35 English under-21 players made appearances in the EPL; the lowest figure since 2005. Despite much conjecture and vociferous debate regarding elite player development within the game, what is clear is that the ever increasing quality of the English Premier League (EPL), underpinned by the extraordinary financial power of elite clubs, has led to a scenario where, to 'break through' to the elite level, young players not only have to be one of the finest in England but also the world. For this reason, the director of youth at the EPL has noted that, "the focus on youth has probably never been as intense or as urgent since the inception of the Premier League as it is right now.” [3].

This notable real world significance is reflected in the increasing amount of research within the sport sciences that has been dedicated to the topic in recent years $[4,5,6,7]$. Notwithstanding the different perspectives taken (e.g., sociological, motor control and learning, stress and coping), this research is ultimately united by a shared focus on the application of scientific principles to help those involved in elite player development. While the development and eventual success of a gifted young player is considered to be influenced by an intricate blend of innate, psychological, and behavioural factors [5], few would dispute that it is also largely shaped by their environmental experience [8]. Indeed, Williams and Reilly [9] contend that the very term "talent development" in football infers that 
Examining the development environments

1 young players are provided with an appropriate learning environment to translate their

2 potential into excellence. Offering support for this view, in a study identifying factors

3 perceived to influence the development of elite academy players [5], providing a rich and

4 vibrant learning environment was considered central to successful progression. In coaching

5 psychology literature, as well as on the topic of developing talent, what constitutes a vibrant

6 learning environment has received increased interest [10]. Traditionally, coaching

7 environments have been criticised for adopting approaches in which young, developing

8 athletes are treated as "sponges", thereby reflecting a more passive style to learning. It is now

9 encouraged that to promote learning, coaches need to encourage active learning (e.g.,

10 questioning methods and problem solving type drills), as well as using strategies to help

11 athletes become aware of their performance (e.g., coach-athlete dialogue) and raise self-

awareness. Highlighting the role of such environments, Mills et al., [5] findings suggested

creating conditions that promote a number of intrapersonal factors associated with success

14 (e.g., resilience, confidence, competitiveness). Indeed, self-awareness was found to be one of

15 the key factors associated with the successful development of Academy football players.

16 Over all, this recognised need to cultivate appropriate developmental climates in which to

17 nurture young players underlines Gagne's [11] contention that exceptional natural abilities

can remain solely as gifts if not effectively nurtured via the developmental process into systematically developed talents. Put simply, elite players would appear to be largely built not born and, as such, points toward the environment created at a youth academy as one of the most directly controllable factors in the life of a young player.

Despite the importance placed on a players' development environment, to-date, little is known about the environments that are created for elite young players. Given that elite youth

24 football in England is considered to be inherently challenging and largely characterised by a highly pressurised climate for success [7], the lack of research in this regard in somewhat 
Examining the development environments

1 surprising. Notwithstanding the contributions of recent investigations [5, 6, 7] that underline

2 the necessity for developing players to be able to cope with the potentially wide-ranging

3 demands they encounter within academy environments; scant research has specifically

4 explored the development environments that are established to nurture players into the elite

5 senior level of the game. In an attempt to redress this imbalance, Mills, Butt, Maynard, and

6 Harwood [12] examined successful elite academy coaches’ perceptions of factors considered

7 to underpin optimal development environments for players on the verge of the professional

8 level. Key themes identified included: (i) espousing a coherent philosophy with clearly

9 defined core values, expectations, and behavioural standards; (ii) promoting whole person

10 development; (iii) empowering key stakeholders (i.e., staff, players, and parents) to create a

11 sense of ownership and relatedness; (iv) forming positive relationships with key stakeholders

12 and prioritising player wellbeing; (v) maintaining well-integrated and stable personnel with

13 strong links to senior team operations (vi); establishing clear and effective lines of

14 communication; (vii) being adaptable and committed to innovation; and (viii) constructing an

15 achievement-focused climate with explicit opportunities to progress. Collectively, the

16 findings emphasised the importance of establishing strong, dynamic organisational cultures

17 within youth academies.

18 Despite the valuable insights generated from this research, our understanding of elite

19 player development environments is far from complete and some important questions remain

20 unanswered. Firstly, obtaining coaches' views of how they shape the environment, while

21 important, only represents one part of the equation. Given that coaching effectiveness is

22 largely considered to be a process-product phenomenon, it would also seem important to

23 elicit the perceptions of developing players to gain their perspectives on the academy

24 environment. Indeed, generating such player-driven insights would help to provide a more

25 complete picture of the environments elite young players are nurtured within. Importantly, 
Examining the development environments

1 insights of this nature would also appear to bridge an evident real-world need. As Green [13,

2 p.10] observed, “despite the time, effort, and huge investment that has gone into England's

3 youth development schemes, one thing the clubs, their leagues, and the FA have not been

4 very good at is finding out what the people who have been through the system felt about it.”

5 Second, despite Williams and Reilly's [9] recommendation that a key area for research

6 in elite youth football is to provide guidelines for nurturing players through each stage of

7 development, few studies, to-date, have specifically focused on key stages along the player

8 development pathway. One such key stage along this pathway relates to the investment years

9 [14]. In talent development terms, this represents the specific transition from elite junior to

10 elite senior where training, competition, and the pursuit of elite level performance become the

11 major foci of a developing athletes' life. In elite youth football terms, this represents the stage

12 where players who show real promise are signed to undertake a two year full-time youth

13 training programme known as an academy scholarship. This structured programme provides

14 young players with a finite window of opportunity to realise their ambition of becoming a

15 professional. Although elite athletes have frequently described this specific transition as the

16 most difficult stage they encountered [15], presently, little is known about players'

17 developmental experiences during this critical period in their early career. This point highlights a gap in the literature that warrants attention. As such, it would seem important for the continued advancement of the area that research begins to build a clear picture of elite development environments as seen through the eyes of players at a pivotal, invariably 'make 21 or break', stage in their footballing lives.

Third, from a methodological perspective, the greater part of talent development research has been retrospective in nature. Specifically, high-achieving elite adult athletes

24 have been asked to reflect on their athletic careers [16]. Notwithstanding these contributions to the literature, research with athletes in situ would seem vital as it may reveal more 
Examining the development environments

1 information about talent development than examining the recalled perspectives of those

2 already at the elite adult level [14]. Indeed, such research would help to identify the positive

3 and negative aspects associated with development so they can either be maximised or

4 minimised in the lives of young athletes [17].

In light of both the empirical and real-world need to better understand the environments

6 in which young players are nurtured, the purpose of the present study was to examine elite

7 academy players' perceptions regarding the quality of their development environment at a

8 decisive stage in their progression to the professional level. It is anticipated that such a

9 detailed, scientific insight will enable a clearer understanding of their current strengths and,

10 importantly, areas that might need improving. In the interests of bridging the gap between

11 research and practice, such information would provide those working within elite youth

12 football with actionable insights that might, in part, help facilitate the development process of

13 gifted young players.

14 METHOD

15 Participants

1650 elite youth football academy players aged $16-18$ years $(m 17.1, \pm s=0.6$ years)

17 participated in the study. Of these, 41 were English and nine were from overseas countries

18 (i.e., African n=3, Asian n=1, Eastern European n=2, Northern European n=3).

19 To capture balanced and geographically diverse perspectives of elite player development

20 environments, players were recruited from academies that were based in the North $(n=1)$,

21 Midlands ( $\mathrm{n}=1)$, and South $(\mathrm{n}=1)$ regions of England. For authenticity of the data, it was also

22 important to recruit a sample that could justifiably be considered elite. To this end, the

23 inclusion of players was based on two strict criteria. First, the players were recruited from

24 academies that participate in the top division of the EPL's Professional Development League

25 (PDL). Academies in this division have been awarded category one status by the EPL which 
Examining the development environments

1 represents the highest tier of elite youth football in England. This category is only awarded to

2 select academies that meet stringent criteria in relation to their youth development

3 programmes [3]. At the time of data collection, only 20 academies held this status. There are

4 approximately 20 scholars (i.e., $1^{\text {st }}$ and $2^{\text {nd }}$ years) in each academy, thus, the present sample

5 equated to approximately 12\% of all elite developing players in England. Second, it was a

6 prerequisite that all players were at the scholarship stage of development (i.e., 16-18 years),

7 and were contracted by the club on a full-time, day-to-day basis. This ensured that reliable,

8 stage-specific perceptions of the development environment could be gathered.

9 Instrumentation : Talent Development Environment Questionnaire (TDEQ)

10 The TDEQ was used to capture the players’ perceptions. Developed by Martindale et al.

11 [1], the TDEQ is a 59 item questionnaire designed to measure the extent to which features of good practice are experienced by athletes in their development environments. Based on key features emanating from the extant talent development literature, the TDEQ has been developed as a generic tool that evaluates the environmental features deemed useful for facilitating development across sports, stage/age, gender, and culture. Specifically, the instrument comprises seven factors: (i) Long-term development focus; (ii) Quality preparation; (iii) Communication; (iv) Understanding the athlete; (v) Support network; (vi) Challenging and supportive environment; and (vii) Long-term development fundamentals. The internal consistency of the questionnaire shows adequate to excellent reliability and a recent validation study also demonstrated robust structural properties and sound ecological validity [1]. In light of its psychometric properties, the TDEQ is considered a tool that can be used with confidence in applied talent development research settings.

Procedure

Following institutional ethics approval, the academy managers of youth academies that met the stipulated inclusion criteria were initially contacted by email detailing the purpose 
Examining the development environments

1 and nature of the study. For those who agreed to participate, convenient times were arranged

2 to collect the data. Before data collection commenced, informed consent was obtained from

3 the academy manager and players which confirmed their understanding of the purpose of the

4 study and their agreement to participate. For those under 18, parental and/or guardian consent

5 was also obtained. Administration of the questionnaires took place at the respective

6 academy's training facilities in quiet classroom conditions under the supervision of the

7 researcher and/or the academy manager and Head of Education and Welfare (HoEW). From

8 the total number of scholars that were invited to participate in the study, 50 completed the

9 survey, indicating an 85\% completion rate. The supervised, on-site, data collection ensured

10 that all questions (i.e., each of the subscales) on the survey had been answered. At the onset,

11 players were also informed that there were no right or wrong answers, given assurances about

12 the confidentiality of their responses, and encouraged to provide honest answers. To further

13 reduce social desirability, the participants were not asked to provide any identifiable details

14 and were assured that any information emanating from the questionnaires would only be

15 displayed as a group average. The questionnaires took approximately 15 minutes to complete.

16 Data analysis

17 Due to the structure of the questionnaire, previous studies [1] using the TDEQ coded responses on a 6-point scale ranging from 1 (strongly agree) to 6 (strongly disagree). For this reason, a lower mean indicated a more favourable perception. However, for ease of

20 interpretation, and in line with Wang and colleagues [18], all items in the present study were

21 coded from 1 (strongly disagree) to 6 (strongly agree). This permitted higher scores to relate

22 to a perception of higher quality experience. ${ }^{1}$

To add validity and accuracy to the interpretation of the data, the reliability of the

24 TDEQ was initially measured using Cronbach’s alpha. Specifically, preliminary statistical

\footnotetext{
${ }^{1}$ Except for negatively phased items where a lower item mean relates to a higher quality perception of that aspect.
} 
Examining the development environments

1 analysis was carried out to determine the internal consistency of the instruments factors. In

2 the present investigation, all but one of the subscale alpha coefficients were found to be

3 adequate and ranged between .60 and .92 (development focus, $\alpha=.92$; quality preparation, $\alpha$

$4=.60$; communication, $\alpha=.84$; understanding of athlete, $\alpha=.63$; support network, $\alpha=.78$;

5 and development fundamentals, $\alpha=.76$ ). Congruent with Wang et al. [18], the challenging

6 and supportive environment factor demonstrated low internal reliability $(\alpha=.40)$, and thus

7 was omitted for interpretation at the subscale level.

8

After establishing reliability, the mean subscale scores were calculated for each of the remaining six factors. Given Martindale et al.’s [1] recommendation to use item scores in conjunction with subscale scores when using the TDEQ in applied research, descriptive statistics were then calculated for all individual item scores within each factor ${ }^{2}$. Following on from this, all items were subsequently quartile ranked by proportion of agreement. This process enabled a detailed, quantitative analysis of the key strengths and areas for improvement as seen through the players' eyes. Items ranked in the top quartile (i.e., top $25^{\text {th }}$ percentile) were greater than $80 \%$ proportion of agreement, and as such were classified as strengths $(+)$ of the development environment. Conversely, items ranked in the bottom quartile (i.e. bottom $25^{\text {th }}$ percentile) were less than $70 \%$ proportion of agreement, and as such were categorised as areas for improvement $(\wedge)$.

\section{RESULTS}

The results are structured in two parts. First, the mean subscale scores of the main variables are displayed (see Table 1) to show how elite player development environments are perceived at an overall factor level. Second, to provide a deeper and more meaningful understanding of the players’ perceptions beyond the subscale scores, each factor is presented in more detail to elucidate trends in the data at an item level. Descriptive statistics for the

\footnotetext{
${ }^{2}$ Nine of the TDEQ's items are miscellaneous and, as such, are not used in calculating the subscale scores, nor have they been included in the item level analysis.
} 
Examining the development environments

1 items within each factor are summarised in Table 2. In addition, Figure 1 displays the specific

2 features of the development environment identified as strengths and areas for improvement.

3 Interpretation of these key areas in light of assumptions derived from the extant talent

4 development literature will be provided in the discussion.

5 Overall

6 At an overall factor level, players reported that their development environment exhibits

7 a long-term development focus, provides robust support networks, and largely demonstrates

8 effective communication. However, features of the environment relating to athlete

9 understanding, long-term development fundamentals, and quality preparation were not

10 viewed as strong. These six factors are discussed in turn below beginning with the three

11 strongest factors.

Long-Term development focus

This factor comprises 27 items that relate to the extent to which development opportunities are specifically designed to facilitate long-term success (e.g., on-going opportunities, rounded development, and clear expectations). The items in this factor also relate to the attitudes, psychological skills, and understanding required for long-term progression (e.g. responsibility, dedication, coping skills). With a mean subscale score of 4.67, this factor emerged as one of the highest performing components of the development environment and, as such, was viewed largely positively by the players.

Within this factor, nearly all players (96\%) agreed to some extent that they were expected to take more responsibility for their own development as they became more experienced $(m=4.83)$. The players also largely indicated that their training was beneficial and challenging $(m=4.91)$; and specifically designed to help them develop in the long term

$24(m=4.85)$. With specific emphasis on the coach, the majority of players reported that their coach cared more about them becoming a professional than having a winning team $(m=4.81)$; 
Examining the development environments

1 constantly reminded them that dedication and desire would be key to how good a performer

2 they would become $(m=5.00)$; was good at making them understand their strengths and

3 weaknesses $(m=4.98)$; and emphasised the need for constant work on fundamental skills ( $m$

$4 \quad$ 4.98). Further, the players mostly agreed that there were people to help them deal with any

5 nerves or worries they experienced $(m=4.60)$; and reported that they were told how they

6 could help each other develop further in their sport $(m=4.85)$. Despite the largely positive

7 responses, approximately two-thirds of players (65\%) agreed to some extent that developing

8 performers are often written off before they have had an opportunity to demonstrate their full

9 potential.

10 Support network

11 This factor comprises eight items that relate to the degree to which a coherent, approachable, and wide-ranging support network is available to help support and develop players in all areas. With a mean subscale score of 4.68 , this factor was one of the highest performing components of the environment. Within this factor, the players revealed that they felt they could pop in to see their coach or support staff whenever they needed to $(m=5.38$ ), and that the coaches and support staff were largely approachable $(m=4.77)$. Players also indicated that they had access to a variety of professional support staff to help their development $(m=4.98) .83 \%$ of players reported that all the different aspects of their development were organised into a realistic schedule; while the majority revealed that their training programmes were specifically developed to their needs $(m=4.51)$. In addition, players largely reported that their coach regularly talks with support staff about what they are trying to achieve $(m=4.68)$; and predominantly considered the coaches and support personnel to be on the same wavelength with what is best for them $(m=4.49)$. The majority of players also revealed that their coach makes sure that their college understands about the demands placed on them with regards training and competition $(m=4.51)$. 
Examining the development environments

\section{Communication}

This factor contains seven items that collectively relate to the degree to which the coach communicates effectively with players in both formal and informal settings. With a mean subscale score of 4.39, this component of the environment performed adequately. Within this factor, the players largely agreed that their coach explained how their training and competition programme work in tandem to help them develop $(m=4.79)$. The majority of players also indicated that they talked with their coach about what current and/or past worldclass performers did to be successful $(m=4.40)$. While $83 \%$ of players agreed to some extent that the feedback they receive almost always relates to their goals; a substantial proportion (45\%) of this agreement was tentative $(m=4.21)$. The players largely reported that they regularly talk with their coach about the things they need to do to progress to the elite senior level $(m=4.49)$; and that they regularly set goals that are tailored to their individual needs ( $m$ =4.49). While the majority of players also indicated that they often discuss the connections between different aspects of their training ( $m=4.51)$; only $44 \%$ either agreed or strongly agreed that they often worked with their coach to identify what their next big test will be $(m=$ 4.15).

\section{Quality preparation}

This factor, consisting of five items, refers to the extent to which clear guidance and opportunities are in place to provide and reinforce quality practice through training, recovery, and competition experiences. With a mean subscale score of 4.16, this factor was viewed as one of the weaker elements of the development environment. All items within this factor were negatively phrased. To this end, a lower mean score relates to a perception of higher quality experiences. Within this factor, the majority of players reported that they get good quality competition experiences at the level they require $(m=2.64)$; and are taught how to balance training, competing, and recovery $(m=2.55)$. However, approximately four out of 
Examining the development environments

1 ten players (39\%) revealed that they felt pressure from their peers to do things differently to

2 what the coach asks $(m=3.04)$; while a similar proportion $(41 \%)$ indicated that they are

3 rarely encouraged to plan for how they would deal with things that might go wrong $(m=$

4 3.06). In addition, only around a half of all players (45\%) reported with certainty (i.e. either

5 agreed or strongly agreed) that the guidelines regarding what they need to do to progress are

6 clear $(m=2.79)$.

$7 \quad$ Understanding the athlete

This factor comprises four items that collectively relate to the extent to which the coach

9 understands the player in depth, at a holistic level, and has developed a strong professional

10 relationship with them. With a mean subscale score of 4.15 , this factor was one of the weaker

11 performing components of the environment. Congruent with the communication factor, all

12 items within this subscale were negatively phrased and, as such, lower item mean scores

13 indicate a more favourable perception. Within this factor, the majority of players felt that

14 their coach took time to talk with other coaches that work with them $(m=2.68)$, and mostly

15 indicated that they received help to develop their mental toughness $(m=2.57)$. However, a

16 third of players (34\%) revealed that their coach rarely talks to them about their well-being ( $m$

17 =3.09); with a similar proportion reporting that their coach seldom takes an interest in their

18 life outside of sport $(m=2.98)$.

\section{Challenging and supportive environment}

This factor consists of four items that relate to the degree to which players are appropriately challenged by, and supported through, their development experiences (e.g., links to higher level players, educational support). Due to an unacceptable alpha coefficient, the mean subscale score was not computed for this factor. At an individual item level, the majority of players reported that they are regularly told that winning and losing "right now" will not determine how successful they will be in the future $(m=4.38)$. In addition, 
Examining the development environments

1 approximately a third (36\%) of players indicated that their college does not support them with

2 their sport when they need it $(m=3.02)$. As well as the largely tentative agreement regarding

3 the opportunities players had to train with players at a level they aspire to $(m=4.02)$; over

4 half (56\%) of all players indicated that they do not often get help from more experienced

5 performers $(m=3.51)$.

$6 \quad$ Long-Term development fundamentals

This factor consists of seven items that collectively relate to the extent to which key

8 features for effective development are embedded in the programme (e.g., on-going

9 opportunities, parental support, and athlete autonomy). With a mean subscale score of 3.95,

10 this factor was the weakest performing facet of the environment. However, it is important to

11 note that this score might have been adversely impacted by an item (i.e., "I am encouraged to

12 participate in other sports”) that is not particularly relevant for the athletes in this study.

13 Within this factor, approximately 4 out of 10 players felt that they would not be given good

14 opportunities if they experienced a dip in performance $(m=3.91)$, while $50 \%$ of players felt

15 that their coach did not make time to talk with their parents about what they are trying to

16 achieve $(m=3.43)$. Moreover, only $47 \%$ of players agreed with conviction that they had

17 opportunities to discuss how more experienced players handled the pressures they face $(m=$

18 4.17). Though the greater part of players indicated that they were involved in most decisions

19 about their development $(m=4.34)$, a fifth $(21 \%)$ reported that they were not. More

20 positively, the majority of players felt that they had their progress and performance reviewed

21 regularly on an individual basis $(m=4.47)$; and largely indicated that the advice their parents

22 provide fits well with the advice they get from their coaches $(m=4.49)$.

DISCUSSION

The purpose of the present study was to examine elite youth football academy players’ 
Examining the development environments

1 progression to the professional level. To our knowledge, the current investigation is the first

2 to reveal the perspectives of such athletes within elite high-performance training

3 environments. As such, the findings offer a step forward in this area, not only within elite

4 youth football, but also elite youth sport in general. The aim of this section is to situate the

5 identified key strengths and areas for improvement (see Figure 1) in light of assumptions

6 derived from the extant talent development literature. Following on from this, we present a

7 number of applied implications and recommendations for those involved in elite player

8 development.

The majority of strengths emerged from the long-term development focus factor and appear to largely relate to coaching practice (e.g., technical instruction, training plans). In doing so, this emphasizes the high quality of coaching players feel they are receiving within elite academies. Given the importance placed on high quality coaching in fostering a rich and vibrant learning environment within football academies [4], this is an encouraging finding. Additional strengths within this factor related to a focus on improvement rather than winning, and the promotion of self-responsibility, both of which are considered key features of effective development environments [12]. The other strong points emerged from the support network factor. Specifically, access to a variety of different professionals (e.g., sport scientists), and the availability of coaches and support staff were both identified as high quality perceptions. Given that well-developed support systems have been shown to be strongly correlated with performance [19], this is an important aspect of the environment that academies are largely perceived to do well.

Highlighting the significance of these aforementioned factors, in a study examining the impact of the development environment on young athletes' goal pursuits and life aspirations, Wang et al. [18] found these factors positively predicted intrinsic goal striving. As such, these 
Examining the development environments

1 motivational climates. Given that elite high-performance environments of this nature might

2 naturally align young players with externally driven goals, this represents a further

3 encouraging finding.

Notwithstanding these positives, the results also revealed a number of lower quality perceptions. As displayed in figure one, these areas for improvement were more evenly

6 spread across the factors, and included issues pertaining to peer pressure, goal-setting,

7 feedback, contingency planning, diminished opportunities due to form, and college/school support. While not overlooking the significance of these areas, three notable themes emerged

9 that specially relate to key factors identified in the literature as exerting a significant

10 influence on player development [12]. As such, we feel these merit particular attention.

11 Specifically, these central themes concerned athlete understanding, links to senior progression, and key stakeholder relationships; each of which will be discussed in turn.

Although understanding the athlete and their world view is considered central to appropriate support [1], this factor was not amongst the best performing components of academy environments. Interestingly, while the aforementioned strengths might be linked to intrinsic drives, a lack of athlete understanding is suggested to promote extrinsic goal striving [18]. This suggests that opposing motivational forces might be at play in academy environments. As previously mentioned, it is conceivable that these extremely competitive, high-performance settings might influence players and coaches towards developing an environment that fosters extrinsic goals and rewards (e.g., successful team, securing a professional contract, getting players through). In such extrinsically motivated climates where players are incessantly scrutinised, and coaches are often under pressure to 'produce'; there exists the potential for coaches to become ego-involved in their work and, in turn, emit controlling behaviours that ultimately promote a controlling climate [20]. It is important to note that in these environments it is likely that coaches are focusing on their primary role, 
Examining the development environments

1 which is to develop players, and ultimately ensure that as many players as possible have the

2 abilities to make it to the professional senior level. Consequently, coaches may be unaware

3 that they are developing extrinsically-driven motivated climates, and thus, indicating a

4 potential area for future coaching education development in Academy football and in other

5 youth sports feeder systems.

6 In addition, while strong support systems are positively linked to performance, a lack of

7 perceived support can lead to poor coping mechanisms and stress [21]. Although high quality

8 perceptions of informational and tangible support emerged as key strengths in this study;

9 perceptions of emotional support were clearly not as strong. Indeed, while players largely

10 indicated that they had good coach-athlete relationships, it was clear that they did not feel

11 particularly understood at a holistic level with coaches seldom expressing an interest in their

12 lives outside of football. Given that young players find themselves immersed in an

13 environment that is fundamentally concentrated on being successful at football, coupled with

14 the tough and masculine culture that tends to characterise professional football, it is not

15 entirely surprising that players' holistic needs might be compromised. To this end, one

16 wonders if these environments might "sow the seed" for an athletic identity and potential

17 identity-foreclosure for these adolescents [22]. If a strong athletic identity is developed,

18 education and the teaching of life skills can often be neglected [23]. To compound matters,

19 such is the strength of many young players' desire to "make it" it is somewhat understandable,

20 despite the limited likelihood of success, why these adolescents might be susceptible to

21 prioritising their football education over their academic and/or general life skill education

22 [24]. Despite the fundamental importance placed by the FA and EPL on player welfare and

23 holistic development; the findings suggest that academies might not be doing enough in this

24 regard. To this end, it would be remiss of academies, both developmentally and ethically, to

25 "gloss over" the socioemotional needs of these individuals, especially given the demands this 
Examining the development environments

1 key stage of development is considered to exert on young athletes. Considering the needs of

2 overseas players provides a cogent example in this regard. Although the present sample was

3 mainly comprised of English and home nation players, a small proportion of the respondents

4 were from overseas. This reflects the increasingly global nature of modern day elite youth

5 football [25]. In addition to the demands placed on local players, overseas players must cope

6 with an often large cultural transition. Indeed, it is highly probable that these players would

7 encounter a range of cultural and lifestyle related issues (e.g., language barrier, home-

8 sickness) that extend far beyond those associated with the football environment. It is likely,

9 therefore, that the demands are heightened for these players in their attempts to adapt to

10 academy life. From from an applied perspective, it would seem important that the

11 psychosocial environments created for these players are well-established to ensure that issues

12 linked to acculturalisation do not have an adverse impact on development.

The second noteworthy theme to emerge in the current study relates to continuing opportunities and links to senior progression. Specifically, players indicated that opportunities to train with senior performers, receiving help from more experienced players, and opportunities to talk about how these players handled the challenges they now face were not readily available. Possibly linked to this, there was a general perception that players are often written off before showing their real potential. These findings might largely be explained by the pervasive short-term "win at all costs” culture that exists in professional football. Indeed, traditionally elite clubs have favoured ready-made, experienced players over 21 youth with a view to having an immediate impact or return on investment. Nevertheless, from a developmental perspective, opportunities for athletes to experience the advanced standard and increased pressure of higher levels (e.g., senior adult, professional) is considered crucial for effective development [1, 5]. Indeed, highlighting the 
Examining the development environments

1 rubric of mental toughness, Mills et al. [5] revealed that challenging training environments

2 (e.g., training with senior team) helped promote key intrapersonal attributes associated with

3 successful progression at this key stage (e.g., resilience, coping with pressure, confidence).

4 As such, it appears crucial that youth and senior team operations are well-integrated. Indeed,

5 any semblance of dichotomy between the two might have serious repercussions for successful

6 player development. In real world operations, it does appear that those professional clubs

7 using well-established players (i.e., those players who are nearing the end of their careers and

8 might be moving towards a youth team coaching role) to bridge this gap, are successfully

9 "getting players through."

10 The third important theme to emerge centred on key stakeholder relationships with

11 specific emphasis on the coach-parent dyad. While some parents are considered to facilitate

12 player development, certain parental behaviours (e.g., conflicting coaching advice) are

13 considered to exert a negative influence [5]. As such, the prevailing view amongst coaches

14 appears to be one that considers parents as more of a hindrance than a help. In support of this

15 notion, the players in the present study indicated that their coach did not make sufficient time

16 to talk with their parents about their development. However, of particular note, players also

17 revealed that their parents' advice was largely congruent with their coaches. These findings

18 suggest that academy coaches might be overlooking the important role parent's play in the

19 development process [16]; even through the investment years where the coach is considered

20 to exert a greater influence [14]. Indeed, forming positive key stakeholder relationships and

21 empowering parents to create a sense of ownership and relatedness are considered key factors

22 underpinning the creation of optimal player development environments and a high-

23 performance culture [12].

24 Applied implications 
Examining the development environments

In the interests of bridging the gap between research and practice, the question remains how academies can be helped to meet the needs of developing players at this decisive stage of development. In this regard, the findings offer a number of practical implications for those involved in elite player development. First, the findings underline the importance of building strong links to the senior team. Although such relationships might ultimately rest upon the senior team manager championing a pro-youth policy, it is recommended that links to senior players could be established in the form of a mentoring scheme. Importantly, rather than inviting $1^{\text {st }}$ year or early career professionals - who may be reluctant to assist players that could be viewed as a threat - such a scheme would lend itself to the participation of already established/late career professionals who may be nearing the end of their careers and looking to transition into coaching. Indeed, we contend that inviting established professionals to pass down their knowledge, share their experiences of the development process, and provide insights into how they met the challenges that young players now face could play a crucially important function in the development of players at this key transitional stage.

$$
\text { Second, it is clear that academies need to pay close attention to the psychosocial }
$$
environments they create for developing players. From a developmental standpoint, this is a serious ethical issue, especially if there is a risk that the nature of these academies might not prepare these individuals for life outside of football. Consequently, we believe those responsible for the design and implementation of academy programmes should not only be mindful of young players' socioemotional needs; but also make a genuine, concerted effort to prepare players for all eventualities. In this regard, for a truly balanced approach to player development, we believe coaches at the youth level should be encouraged to ground their practice around an athlete-centred model where performance excellence co-exists in the same environment as personal excellence. When applied, this approach to coaching is considered a powerful tool in empowering young athletes to learn and take more responsibility for their 
Examining the development environments

1 own development, which ultimately, results in enhanced performance and a thriving,

2 supportive team environment [26].

Notwithstanding the importance of what essentially rests at the heart of the coaches' role (i.e., technical instruction), in light of the players’ perceptions, we feel an athlete-centred approach would be more developmentally appropriate. We also acknowledge that some readers may be cynical that such utopia is possible given the culture of the game. Certainly,

7 the efficacy of such an approach would greatly rest upon the club advocating a holistic policy

8 as part of their vision for player development. However, if the conditions can be created

9 whereby an academy manager feels secure in the knowledge that producing players is not the sole outcome measure, we are confident that such a model of development could be successfully woven into the fabric of an academy’s culture.

The findings also offer a number of applied implications for sport psychologists working in youth football settings. Specifically, the nature of these implications would involve practitioners going beyond the traditional canon of mental skills training, as well as performance enhancement techniques. For example, to overcome the influence of a largely externally driven sport culture that is susceptible to the establishment of controlling climates, sport psychologists could have an important role to play in the promotion of autonomysupportive coaching behaviours. Such coaching is considered to make players feel more competent in their sport, more autonomous in their actions, and better related to significant others from their environment [27]. Enhanced perceptions of these three basic psychological needs help foster more intrinsic drives and adaptive goal orientations that are considered fundamental to optimum functioning and positive self-growth [28]. important that academies strive to build more positive working relationships with parents. To help accomplish this, we feel sport psychologists would be ideally placed to facilitate parent 
Examining the development environments

1 workshops geared towards optimising their influential role as a football parent. In light of the

2 suggestion that the intensive journey of an academy player is mirrored by an equally

3 demanding journey for their parents [29], these workshops, could play a key function in

4 parental development by also acting as an organised forum for parents to share their

5 experiences.

6 Strengths and limitations

7

From a real-world perspective, a primary strength of this investigation is that a detailed,

8 scientific attention to elite players' perceptions regarding the quality of their talent

9 development environment might assist academies to optimise their programmes. In addition,

10 as a preliminary attempt to examine elite football academy players’ perceptions of the quality

11 of their development environment at a key stage in their journey to the professional level, the

12 findings not only advance our limited understanding regarding talent development

13 environments; but also help to bridge an important gap in the knowledge base regarding key

14 stages of athletic development. Given the high quality sample, we also feel this investigation

15 was enhanced by ensuring accurate and reliable perceptions of elite high-performance

16 environments could be assembled. From a methodological viewpoint, by capturing "in the

17 moment” views of elite players presently involved in the talent development process on a

18 day-to-day basis, an additional strength of the study involved overcoming some of the

19 limitations of previous retrospective designs. Moreover, given the recommendation to

20 consider individual items as well as overall factor scores when using the TDEQ in applied

21 research, the present study was enriched by 'drilling down' to an item level which enabled

22 more meaningful and practical insights.

Notwithstanding these strengths, some limitations must also be acknowledged. Firstly,

24 given the culturally specific focus on the English academy system, the transferability of our

25 interpretations to player development environments in other countries is speculative. As such, 
Examining the development environments

1 readers should be circumspect in any attempt to relate the findings to other contexts. Second,

2 as the TDEQ has been designed as a generic tool, its developers recognise that a range of

3 context-specific issues may be apparent which might necessitate the development of sport

4 and/or stage specific versions [1]. Given the complex, idiosyncratic sub-culture of elite youth

5 football, we concur with this suggestion. Indeed, to fully evaluate the experiences of

6 developing players, a sport-specific diagnostic tool unique to the sporting population might

7 need to be developed, which we feel would have far greater precision as an applied diagnostic

8 tool. To illustrate our point, although cross training or participating in other sports is

9 considered important for athletes in the sampling years of development, this item would not

10 be appropriate for those in the investment stage. However, in the TDEQ’s current form,

11 disagreeing with this statement would relate to a low quality perception of the environment,

12 and as such might influence the reliability of the overall subscale score. Despite this potential

13 limitation, given the robust questionnaire development process, ensuing level of

14 psychometric properties, and sound ecological validity, we are confident in the insights

15 generated by the current instrument.

16 Concluding remarks

Although academies were generally viewed positively, the findings suggest that these

19 elite high-performance environments might not be fully meeting young players'

20 developmental needs. Indeed, given that player welfare, links to senior progression, and

21 positive key stakeholder relationships are all suggested to be vital for the creation and

22 regulation of an optimal development environment, it would seem imperative that academies

23 pay closer attention to these potentially problematic areas. Encouragingly, as elite players are

24 largely considered to be "built" not "born”, the academy environment would appear to be one

25 of the most directly controllable factors in the life of a young player. To this end, we have put

26 forward a number of suggestions for how these areas might, in part, be addressed. From a 
Examining the development environments

1 broader talent development perspective, there remains a clear need for substantiated,

2 evidence-based practice concerning the creation of optimal talent development environments.

3 With a particular emphasis on football, establishing such environments would appear crucial

4 if the FA and Premier League wish to realise their aspirations of improving current youth

5 development programmes. While we believe the present study represents a step forward in

6 achieving that goal, it is imperative for future research to continue to determine the key

7 processes and mechanisms that underpin effective player development with a view to

8 bridging the gap between research and practice, and ultimately helping young players

9 transform their gifts into systematically developed talents.

References

11 [1] Martindale, R. J. J., Collins, D., Douglas, C. and Whike, A., Examining the Ecological

12 Validity of the Talent Development Environment Questionnaire, Journal of Sports Sciences,

13 2012, 31, 41-47.

14 [2] Magowan, G., Premier League: English under-21s Reaches New Low, www.bbc.co.uk

15 (web page accessed on 06/06/2013).

16 [3] The Premier League., Elite Player Performance Plan: Long-term Strategy Designed to

17 Advance Premier League Youth Development, http://www.premierleague.com (web page

18 accessed on 25/08/2012).

19 [4] Cushion, C., Ford, P.R. and Williams, M.A., Coach Behaviours and Practice Structures in

20 Youth Soccer: Implications for Talent Development, Journal of Sports Sciences, 2012, 30,

$21 \quad 1631-1641$.

22 [5] Mills, A., Butt, J., Maynard, I. and Harwood, C., Identifying Factors Perceived to

23 Influence the Development of Elite Youth Football Academy Players, Journal of Sport

24 Sciences, 2012, 30, 1593-1604. 
Examining the development environments

1 [6] Reeves, C. W., Nicholls, A. R. and McKenna, J., Stressors and Coping Strategies Among

2 Early and Middle Adolescent Premier League Academy Football Players: Differences

3 According to Age, Journal of Applied Sport Psychology, 2009, 21, 31-48.

4 [7] Sagar, S. S., Busch, B.K. and Jowett, S., Success and Failure, Fear of Failure, and Coping

5 Responses of Adolescent Academy Football Players, Journal of Applied Sport Psychology,

$6 \quad 2010,22,213-230$.

7 [8] Reilly, T., Williams, A. M. and Richardson, D., Identifying talented players, in: Reilly, T.

8 and Williams, A.M., eds., Science and Soccer II, London, Routledge, 2003, 307-326.

9 [9] Williams, A. M. and Reilly, T., Talent Identification and Development in Soccer,

10 Journal of Sports Sciences, 2000, 18, 657-66.

11 [10] Gilbert, W., Introduction to Special Issue: Coach Education. The Sport Psychologist,

12 2006, 20, 123-125.

13 [11] Gagné, F., Transforming Gifts into Talents: The DMGT as a Developmental Theory, in:

14 Colangelo, N. and Davis, G.A., eds., Handbook of Gifted Education, 3rd edn., Boston, Allyn

15 and Bacon, 2003, 60-74.

16 [12] Mills, A., Maynard, I.W., Butt, J. and Harwood, C., Creating Effective Development

17 Environments within Elite English Football Academies, The Sport Psychologist, 2013, In

18 press.

19 [13] Green, C., Every Boy's Dream: England's Football Future on the Line, London, A and C 20 Black Publishers Ltd, 2009.

21 [14] Côté, J., The Influence of the Family in the Development of Talent in Sports, The Sport 22 Psychologist, 1999, 13, 395-417.

23 [15] Stambulova, N., Alfermann, D., Statler, T. and Côté, J., Career Development and

24 Transitions of Athletes: The ISSP Position Stand, International Journal of Sport and

25 Exercise Psychology, 2009, 7, 395-412. 
Examining the development environments

1 [16] Gould, D., Dieffenbach, K. and Moffett, A., Psychological Characteristics and Their

2 Development in Olympic Champions, Journal of Applied Sport Psychology, 2002, 14, 172-

3204

4 [17] Durand-Bush, N. and Salmela, J. H., The Development and Maintenance of Expert

5 Athletic Performance: Perceptions of World and Olympic Champions, Journal of Applied

6 Sport Psychology, 2002, 14, 154-171.

7 [18] Wang, C.K.J., Sproule, J., McNeill, M., Martindale, R. J. J. and Lee, K.S., Impact of

8 The Talent Development Environment on Achievement Goals and Life Aspirations in

9 Singapore, Journal of Applied Sport Psychology, 2011, 23, 263-276.

10 [19] Rees, T., Influence of Social Support on Athletes, in: Jowett, S. and Lavallee, D., eds.,

11 Social Psychology in Sport, Champaign, IL, Human Kinetics, 2007, 223-231.

12 [20] Mageau, G.A. and Vallerand, R.J., The Coach-Athlete Relationship: A Motivational

13 Model, Journal of Sports Sciences, 2003, 21, 883-904.

14 [21] Lafferty, M. E. and Dorrell, K., Coping Strategies and the Influence of Perceived

15 Parental Support in Junior National Age Swimmers, Journal of Sports Sciences, 2006, 24, $16 \quad 253-259$.

17 [22] Murphy, G. M., Petitpas, A. J. and Brewer B. W., Identity Foreclosure, Athletic Identity, 18 and Career Maturity in Intercollegiate Athletes, The Sport Psychologist, 1996, 10, 239- 246.

19 [23] Grove, A. R., Lavallee, D. and Gordon, S., Coping with Retirement from Sport: The 20 Influence of Athletic Identity, Journal of Applied Sport Psychology, 1997, 9, 191-203.

21 [24] Platts, C. and Smith, A., The Education and Welfare of Young People in Professional

22 Football Academies in England: Some Implications of The White Paper on Sport,

23 International Journal of Sport Policy, 2009, 1, 323-39. 
1 [25] Nesti, M., Littlewood, M., O’Halloran, L., Eubank, M. and Richardson, D., Critical

2 Moments in Elite Premiership Football: Who Do You Think You Are? Physical Culture and

3 Sport Studies and Research, 2012, 56, 23-32.

4 [26] Kidman, L., Athlete-Centred Coaching: Developing Inspired and Inspiring People,

5 Christchurch: Innovative Print Communications Ltd, 2005.

6 [27] Álvarez, M. Balaguer, I. Castillo, I. and Duda J., Coach Autonomy Support and Quality

7 of Sport Engagement in Young Soccer Players, The Spanish Journal of Psychology, 2009, 2,

$8 \quad 138-148$.

9 [28] Deci, E. L. and Ryan, R. M., Intrinsic Motivation and self-Determination in Human

10 Behavior, New York, Plenum, 1985.

11 [29] Harwood, C., Drew, A. and Knight, C. J., Parental Stressors in Professional Youth

12 Football Academies: A Qualitative Investigation of Specialising Stage Parents, Qualitative

13 Research in Sport and Exercise, 2010, 2, 39 -55. 
Examining the development environments

Table I. Mean subscale scores for player perceptions of the quality of the development environment

\begin{tabular}{lcc}
\hline TDEQ Subscales & $\mathrm{M}$ & $\mathrm{SD}$ \\
& & \\
\hline & & \\
Long-Term Development Focus & 4.72 & 0.87 \\
Quality Preparation & 4.18 & 1.11 \\
Communication & 4.43 & 0.92 \\
Understanding the Athlete & 4.17 & 1.13 \\
Support Network & 4.73 & 0.92 \\
Long-Term Development Fundamentals & 3.98 & 1.10
\end{tabular}


Table II Means and standard deviations for TDEQ items within each factor

\section{Factor 1: Long-Term Development Focus}

My coach is good at helping me to understand my strengths \& weaknesses

My coach is good at helping me understand what I'm doing \& why I'm doing it
My coach emphasises the need for constant work on fundamental skills

$4.85 \quad 0.88$

The more experienced I get the more my coach encourages me to take responsibility for my own development

My development plan incorporates a variety of physical preparation

If I got injured I believe I would continue to receive a good standard of support

I am constantly reminded that my personal dedication \& desire will be key to how good a performer I become

My coach constantly reminds me what he expects of me

My coach is a positive supporting influence on me

My coaches care more about helping me to become a professional than they do about having a winning team right now

My coach plans training to incorporate a wide variety of useful skills \& attributes

My training is specifically designed to help me develop effectively in the long term

My coach emphasises that what I do in training \& competition is far more important than winning

I am being trained to be ready for almost anything that is thrown at me in sport \& life

I spend most of my time developing skills \& attributes that my coach tells me I will need to compete at the pro level

My training sessions are normally beneficial \& challenging

Me \& my sports mates are told how we can help each other develop further in the sport

My coach allows me to learn through making my own mistakes

I am encouraged to keep perspective by balancing frustrations in one area with thinking about good progress in others

Organisation is a high priority to those who develop my training programme

There are people who help me/teach me how to deal positively with any nerves or worries that I experience

If it didn't work out for me here, there are other good opportunities that would help me to keep progressing

Developing performers are often written off before they have had a chance to show their real potential*

My coaches and those who support me give me straight answers to my questions

\section{Factor 3: Communication}

I regularly set goals with my coach that are specific to my individual development

My coach \& I regularly talk about things I need to do to progress to the top level

My coach often talks to me about the connections/overlap between different aspects of my training

My coach \& I talk about what current \&/or past world-class performers did to be successful

My coach and I often try to identify what my next big test will be before it happens

My coach explains how my training \& competition programme work together to help me develop

Feedback I get from my coaches almost always relates directly to my goals

My coach doesn't appear to be that interested in my life outside of sport*

My coach rarely takes the time to talk to other coaches who work with me*

I don't get much help to develop my mental toughness in sport effectively*

Factor 5: Support Network

I have access to a variety of different professionals to help my development

I can pop in to see my coach or other support staff whenever I need to

My coaches talk regularly to the other people who support me in my sport about what I'm trying to achieve

My training programmes are developed specifically to my needs

My coaches ensure that my college understands about me \& my training/comp

Those who help me in my sport seem to be on the same wavelength when it comes to what is best for me

\section{Factor 6: Challenging \& Supportive Environment}

\section{My school/college doesn't really support me with my sport when I need it*}

I am regularly told that winning and losing just now does not indicate how successful I will be in the future

I have the opportunity to train with performers who are at a level I'm aspiring to

I don't often get any help from more experienced performers*

\section{Factor 7: Long-Term Development Fundamentals}

I would be given good opportunities even if I experienced a dip in performance

I am encouraged to participate in other sports and/or cross train

I often have the opportunity to talk about how more experienced performers have handled the challenges I face 
Challenging \& Supportive Environment

$(\wedge)$ Support from school/college when needed

$(\wedge)$ Help from experienced players

$(\wedge)$ Opportunities to train with players at a level they aspire to

\section{Communication}

$(\wedge)$ Identifying next big test before it happens

$(\wedge)$ Providing feedback relating directly to players' goals

\section{Quality Preparation}

$(\wedge)$ Encouraging players to plan for how to deal with things that might go wrong

$(\wedge)$ Providing clear guidelines regarding what players need to do to progress

$(\wedge)$ Peer pressure to do things differently from what coaches ask

\section{Support Network}

(+) Access to a variety of different professionals to help support development

$(+)$ Coaches \& support staff always available if required

Understanding the Athlete

$(\wedge)$ Interest shown in life outside of football

(^) Talking with players about their well-being

\section{Long Term Development Focus}

$(+)$ Care more about becoming a pro than having a winning team

(+) Variety of physical preparation built into development plan

$(+)$ Would continue to receive a good standard of support if injured

(+) Help to understand strengths \& weaknesses/what \& why they do things

(+) Told how they can help each other develop further

$(+)$ Encouraged to take responsibility for own development

$(+)$ Beneficial \& challenging training sessions/incorporates variety of useful skills

$(+)$ Encouraged to constantly work on fundamental skills

$(+)$ Reminded that dedication \& desire is central to success

$(+)$ Training specifically designed to help develop in the long-term

$(+)$ Strong focus on developing skills \& attributes needed at the pro level

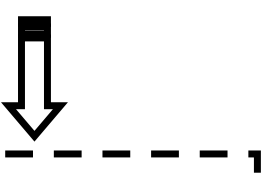

Elite Player

I

Development I

Environments

\section{1}

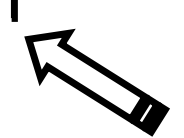

Long Term Development

Fundamentals

(^) Providing opportunities despite dip in performance

$(\wedge)$ Opportunities to talk about how more experienced players handled the challenges they face

(^) Discussing development \& ambitions with parents

$(\wedge)$ Players often written off before showing full potential

Figure 1. Features of elite player development environments perceived as key strengths $(+)$ and key areas for improvement $(\wedge)$. Note: For the purposes of calculating sub-scale scores and ranking proportion of agreement, all negatively phrased item raw-scores were reverse coded. 